TRANSACTIONS OF THE

AMERICAN MATHEMATICAL SOCIETY

Volume 358, Number 2, Pages 743-757

S 0002-9947(05)03822-5

Article electronically published on March 18, 2005

\title{
ISOVARIANT BORSUK-ULAM RESULTS FOR PSEUDOFREE CIRCLE ACTIONS AND THEIR CONVERSE
}

\author{
IKUMITSU NAGASAKI
}

\begin{abstract}
In this paper we shall study the existence of an $S^{1}$-isovariant map from a rational homology sphere $M$ with pseudofree action to a representation sphere $S W$. We first show some isovariant Borsuk-Ulam type results. Next we shall consider the converse of those results and show that there exists an $S^{1}$-isovariant map from $M$ to $S W$ under suitable conditions.
\end{abstract}

\section{INTRODUCTION}

The original Borsuk-Ulam theorem proved by 1 states that if there is a continuous map $f: S^{m} \rightarrow S^{n}$ so that $f(-x)=-f(x)$ for all $x \in S^{m}$, then $m \leq n$ holds (and as easily seen, the converse is true as well). For over seventy years this theorem was generalized in various directions and applied in several fields of mathematics (cf. [13, 18, 19]). In the viewpoint of the equivariant topology, Borsuk-Ulam type theorems are deeply concerned with the problem of whether or not a $G$-equivariant map exists between given $G$-spaces. Recently one of such Borsuk-Ulam type results played a significant role in a partial solution of the 11/8-conjecture [10].

On the other hand, in the equivariant topology, there are various theories and results in the isovariant setting, e.g., (isovariant) surgery theory on stratified sets [3], [7, isovariant homotopy theory [8, the isovariant $s$-cobordism theorem [3], 12, Theorem 4.42], etc.

In this paper we shall study Borsuk-Ulam type results and their converse in the isovariant setting. A $G$-equivariant map $f: X \rightarrow Y$ is called $G$-isovariant if $f$ preserves the isotropy groups, i.e., $G_{x}=G_{f(x)}$ for all $x \in X$. (Throughout this paper all maps are assumed to be continuous.) Let $\operatorname{Iso}(X)$ denote the set of isotropy groups of a $G$-space $X$. In [20] and [15], isovariant variants of the BorsukUlam theorem were studied, which provide nonexistence results of isovariant maps between representation spheres. We shall consider a similar problem in the following situation: $G$ is the circle group $S^{1}$, the target space is a representation sphere, and the source space is a rational homology sphere $M$ with effective smooth $S^{1}$-action of which singular set $M_{s}:=\bigcup_{H \neq 1} M^{H}$ is empty or of $\operatorname{dim} M_{s} / S^{1}=0$. We call such an action the discrete singularity action or the DS-action for short. A rational homology sphere means a smooth closed manifold of which homology groups with rational coefficients are isomorphic to those of $S^{m}, m=\operatorname{dim} M$. As is easily seen,

Received by the editors March 1, 2004.

2000 Mathematics Subject Classification. Primary 55M20; Secondary 57S15, 55M25, 55S35.

Key words and phrases. Isovariant map, Borsuk-Ulam theorem, pseudofree action, multidegree, Hopf theorem, obstruction theory.

The author was partially supported by Grant-in-Aid for Scientific Research.

(c)2005 American Mathematical Society Reverts to public domain 28 years from publication 
discrete singularity $S^{1}$-actions on a connected closed orientable manifold $M$ are divided into three types:

Type F: $M_{s}$ is empty, namely, $S^{1}$ acts freely on $M$.

Type SF: $M_{s}$ is nonempty and consists of finitely many isolated fixed points, namely, $S^{1}$ acts semifreely on $M$ with isolated fixed points.

Type PF: $M_{s}$ is nonempty and consists of finitely many exceptional orbits (i.e., the isotropy groups of nonfree orbits are nontrivial finite groups), which is just the pseudofree action in the sense of Montgomery and Yang [14] or Petrie [17.

Note that there is no mixed type of $\mathrm{SF}$ and $\mathrm{PF}$, in fact $\operatorname{dim} M$ is even in type $\mathrm{SF}$ and odd in type $\mathrm{PF}$, since the slice representation of a fixed point or a singular orbit has even dimension. In section 1 we shall show the following isovariant Borsuk-Ulam type results.

Theorem A. Let $M$ be an $S^{1}$-rational homology sphere of type $F, S F$ or $P F$, and let $S W$ be the representation sphere of an orthogonal $S^{1}$-representation $W$. If an $S^{1}$-isovariant map $f: M \rightarrow S W$ exists, the following inequalities hold: In type $F$,

(F): $\operatorname{dim} M+1 \leq \operatorname{dim} S W-\operatorname{dim} S W^{H}$ for $1 \neq H \leq S^{1}$.

In type $S F$,

(SF): $\operatorname{dim} M \leq \operatorname{dim} S W-\operatorname{dim} S W^{H}$ for $1 \neq H \leq S^{1}$.

In type $P F$,

(PF1): $\operatorname{dim} M-1 \leq \operatorname{dim} S W-\operatorname{dim} S W^{H}$ for $H \neq 1$ such that $H \leq C$ for some $C \in \operatorname{Iso}(M)$, and

(PF2): $\operatorname{dim} M+1 \leq \operatorname{dim} S W-\operatorname{dim} S W^{H}$ for $H \neq 1$ such that $H \not \leq C$ for any $C \in \operatorname{Iso}(M)$.

Here if $S W^{H}$ is empty, then we set $\operatorname{dim} S W^{H}=-1$ by convention. In sections 24, we shall investigate the converse of Theorem A using equivariant obstruction theory. Our results on the converse are summarized as follows:

Theorem B. Let $M$ and $S W$ be as above. Suppose that $\operatorname{Iso}(M) \subset \operatorname{Iso}(S W)$. In each type, if the corresponding inequalities hold, then there exists an $S^{1}$-isovariant map from $M$ to $S W$.

Remark. The condition $\operatorname{Iso}(M) \subset \operatorname{Iso}(S W)$ is obviously necessary.

In section 2 we shall prove Theorem $\mathrm{B}$ in types $\mathrm{F}$ and $\mathrm{SF}$ by showing that the obstruction groups vanish. In the case of type PF, the obstruction groups do not necessarily vanish, hence we need to detect the obstruction class itself. To do that, in section 3, we introduce the multidegree of certain $S^{1}$-maps and show some Hopf type results. In section 4 we shall prove Theorem B in type PF in the following way. Let $N_{i}$ be closed $S^{1}$-tubular neighborhoods of the exceptional orbits. Set $N=\coprod_{i} N_{i}$ and $X=M \backslash(\operatorname{int} N)$. We first construct isovariant maps $f_{i}: N_{i} \rightarrow S W$ and next discuss the extendability of $\coprod_{i} f_{i \mid \partial N_{i}}: \partial X \rightarrow S W$ to an isovariant map from $M$ using the multidegree. The essential point is that the obstruction vanishes for suitable choices of $f_{i}$.

In section 5, we shall illustrate some examples, in particular, we shall give an answer to (an analogue of) the question posed in [16]. Let $T_{n}, n \in \mathbb{Z}$, denote the irreducible complex representation (space) of $S^{1}$ given by $\rho_{n}: S^{1} \rightarrow S U(1)\left(=S^{1}\right)$, $z \mapsto z^{n}$. 
Example. Let $p, q, r$ be pairwise coprime integers greater than 1 . There exists an $S^{1}$-isovariant map

$$
f: S\left(T_{p} \oplus T_{q} \oplus T_{r}\right) \rightarrow S\left(T_{1} \oplus T_{p q} \oplus T_{q r} \oplus T_{r p}\right) .
$$

\section{Some isovariant Borsuk-Ulam type Results}

We begin by recalling well-known Borsuk-Ulam type results. Let $G$ be a compact Lie group, and let $S V$ and $S W$ be representation spheres with free $G$-action. A Borsuk-Ulam type theorem states that if there is a $G$-map from $S V$ to $S W$, then the inequality $\operatorname{dim} S V \leq \operatorname{dim} S W$ holds (see for example [6]). This result is generalized by many authors, for example, from [4, Theorem 6.4] the following generalization can be derived.

Proposition 1.1. Let $X$ and $Y$ be finite-dimensional $S^{1}-C W$ complexes with finitely many isotropy groups and without $S^{1}$-fixed points (but not necessarily free). Suppose $H_{*}(X ; \mathbb{Q}) \cong H_{*}\left(S^{m} ; \mathbb{Q}\right)$ and $H_{*}(Y ; \mathbb{Q}) \cong H_{*}\left(S^{n} ; \mathbb{Q}\right)$. If there is an $S^{1}-$ map from $X$ to $Y$, then $m \leq n$ holds.

Isovariant variants of the Borsuk-Ulam theorem were first studied in [20] (see also [15]). One of them is stated as follows:

Proposition 1.2. Suppose that $G$ is solvable. If there is a $G$-isovariant map from $S V$ to $S W$, then the inequality

$$
\operatorname{dim} S V-\operatorname{dim} S V^{G} \leq \operatorname{dim} S W-\operatorname{dim} S W^{G}
$$

holds.

These Borsuk-Ulam type theorems provide some dimensional conditions for the existence of an isovariant map. We first consider an $S^{1}$-rational homology sphere $M$ of type F, namely $S^{1}$ acts freely on $M$. Let $f: M \rightarrow S W$ be an $S^{1}$-isovariant map. By isovariance, $f$ maps $M$ into $S W \backslash S W^{H}$ for $1 \neq H \leq S^{1}$. Since $S W \backslash S W^{H}$ is $S^{1}$-homotopy equivalent to $S\left(W^{H}\right)^{\perp}$, where $S\left(W^{H}\right)^{\perp}$ denotes the unit sphere of the subrepresentation defined as the orthogonal complement $\left(W^{H}\right)^{\perp}$ of $W^{H}$ in $W$ (i.e., $\left.W=W^{H} \oplus\left(W^{H}\right)^{\perp}\right)$, there is an $S^{1}$-map $\bar{f}: M \rightarrow S\left(W^{H}\right)^{\perp}$. By Proposition 1.1 one can see

$$
\operatorname{dim} M \leq \operatorname{dim} S\left(W^{H}\right)^{\perp}=\operatorname{dim} S W-\operatorname{dim} S W^{H}-1 .
$$

Thus we obtain:

Proposition 1.3. Let $M$ be an $S^{1}$-rational homology sphere of type $F$. If there is an $S^{1}$-isovariant map $f: M \rightarrow S W$, then

(F): $\operatorname{dim} M+1 \leq \operatorname{dim} S W-\operatorname{dim} S W^{H}$ for $1 \neq H \leq S^{1}$.

We next consider the case of type SF. By definition $S^{1}$ acts semifreely on $M$ with isolated fixed points. Let $x \in M^{S^{1}}$ and let $f: M \rightarrow S W$ be an $S^{1}$-isovariant map. By the slice theorem (see [2], [11]), there is a (small) invariant neighborhood $N$ of $x$ which is $S^{1}$-diffeomorphic to some representation disk $D V$. Restricting $f$ to $\partial N \cong S V$, we have an $S^{1}$-isovariant map $\bar{f}: S V \rightarrow S W$. Since $S^{1}$ acts freely on $S V$, it follows from Proposition 1.2 that $\operatorname{dim} S V+1 \leq \operatorname{dim} S W-\operatorname{dim} S W^{H}$ for $1 \neq H \leq S^{1}$. Thus we obtain the following proposition. 
Proposition 1.4. Let $M$ be of type $S F$. If there is an $S^{1}$-isovariant map $f: M \rightarrow$ $S W$, then

(SF): $\operatorname{dim} M \leq \operatorname{dim} S W-\operatorname{dim} S W^{H}$ for $1 \neq H \leq S^{1}$.

Remark. In this proposition, $M$ does not need to be a rational homology sphere.

Remark. Inequalities (F) and (SF) are equivalent to the following conditions, respectively.

$\left(\mathbf{F}^{\prime}\right): \operatorname{dim} M+1 \leq \operatorname{dim} S W-\operatorname{dim} S W_{s}$.

$\left(\mathbf{S F}^{\prime}\right): \operatorname{dim} M \leq \operatorname{dim} S W-\operatorname{dim} S W_{s}$.

Here $S W_{s}$ denotes the singular set of $S W$, i.e., $S W_{s}=\bigcup_{H \neq 1} S W^{H}$, and $\operatorname{dim} S W_{s}=$ $\max \left\{\operatorname{dim} S W^{H} \mid H \neq 1\right\}$.

In the case of type PF, we can see the following.

Proposition 1.5. Let $M$ be an $S^{1}$-rational homology sphere of type PF. If there is an $S^{1}$-isovariant map $f: M \rightarrow S W$, then

(PF1): $\operatorname{dim} M-1 \leq \operatorname{dim} S W-\operatorname{dim} S W^{H}$ for $H \neq 1$ such that $H \leq C$ for some $C \in \operatorname{Iso}(M)$, and

(PF2): $\operatorname{dim} M+1 \leq \operatorname{dim} S W-\operatorname{dim} S W^{H}$ for $H \neq 1$ such that $H \not \leq C$ for any $C \in \operatorname{Iso}(M)$.

Proof. Let $H$ be a nontrivial finite subgroup of $S^{1}$ such that $H \leq C$ for some $C \in \operatorname{Iso}(M)$. Take an exceptional orbit isomorphic to $S / C$ in $M$. By the slice theorem its (closed) $S^{1}$-tubular neighborhood $N$ has the form of $S^{1} \times{ }_{C} D U$ for some $C$-representation $U$. Since $S^{1}$ acts freely on the outside of (1-dimensional) exceptional orbits, $C$ acts freely on $S U$, and $\operatorname{dim} S U=\operatorname{dim} M-2$. Restricting $f$ to $S U$, we have a $C$-isovariant map $\bar{f}: S U \rightarrow S W$, and by restricting the action, $\bar{f}$ is regarded as an $H$-isovariant map. It follows from Proposition 1.2 that

$$
\operatorname{dim} S U+1 \leq \operatorname{dim} S W-\operatorname{dim} S W^{H},
$$

which shows (PF1): $\operatorname{dim} M-1 \leq \operatorname{dim} S W-\operatorname{dim} S W^{H}$. Let $H$ be a nontrivial finite subgroup of $S^{1}$ such that $H \not \subset C$ for any $C \in \operatorname{Iso}(M)$. Then, one can see that $f$ maps $M$ into $S W \backslash S W^{H}$. By the same argument as in Proposition 1.3 we obtain (PF2): $\operatorname{dim} M+1 \leq \operatorname{dim} S W-\operatorname{dim} S W^{H}$.

Remark. In the proof of (PF1), $M$ does not need to be a rational homology sphere.

Thus the proof of Theorem A is complete.

\section{EXISTENCE OF ISOVARIANT MAPS IN TYPES F AND SF}

In this section we shall discuss the converse of Theorem A in the cases of types $\mathrm{F}$ and SF. The main tool is equivariant obstruction theory as described in [5, II 3]. For convenience we recall necessary notations and facts. Let $(X, A)$ be a relative $G$-CW complex such that $G$ acts freely on $X \backslash A$. Denote by $X_{n}$ the $n$-skeleton of $(X, A)$ and by $C_{*}(X, A)\left(=H_{*}\left(X_{*}, X_{*-1} ; \mathbb{Z}\right)\right)$ the cellular chain complex. The $G$-action on $X$ induces a $G$-action on $C_{*}(X, A)$ and the identity component $G_{0}$ acts trivially on $C_{*}(X, A)$. Therefore $C_{*}(X, A)$ inherits a (left) $\mathbb{Z}\left(G / G_{0}\right)$-module structure. Taking a $\mathbb{Z}\left(G / G_{0}\right)$-module $\pi$ as coefficients, we define the (equivariant) cochain complex $C_{G}^{*}(X, A ; \pi)$ to be $\operatorname{Hom}_{\mathbb{Z}\left(G / G_{0}\right)}\left(C_{*}(X, A), \pi\right)$. We denote by $\mathfrak{H}_{G}^{*}(X, A ; \pi)$ its cohomology group. Note [5, p.112] that there is an isomorphism

$$
\mathfrak{H}_{G}^{*}(X, A ; \pi) \cong H^{*}(X / G, A / G ; \pi) .
$$


(Here $H^{*}(X / G, A / G ; \pi)$ is a cohomology group with local coefficients in general.) Let $Y$ be a path-connected $n$-simple $G$-space, $n \geq 1$. The homotopy group $\pi_{n}(Y)$ $\left(=\left[S^{n}, Y\right]\right)$ inherits a $\mathbb{Z}\left(G / G_{0}\right)$-module structure. Obstruction theory asserts that the obstruction to an extension of a $G$-map $f: A \rightarrow Y$ to $F: X \rightarrow Y$ lies in $\mathfrak{H}_{G}^{*}\left(X, A ; \pi_{*-1}(Y)\right)$, and the obstruction to the existence of an equivariant homotopy between extensions $F$ and $F^{\prime}$ of $f$ lies in $\mathfrak{H}_{G}^{*}\left(X, A ; \pi_{*}(Y)\right)$.

Let $S W$ be an $S^{1}$-representation sphere with effective action, i.e., $W$ is a faithful representation. This is also equivalent to being $S W \backslash S W_{s} \neq \emptyset$. Set $Y=S W \backslash S W_{s}$, and let $\mathcal{A}$ be the set of isotropy groups $H$ of $S W$ with $\operatorname{dim} S W^{H}=\operatorname{dim} S W_{s}$.

Lemma 2.1. Let $W$ be a faithful $S^{1}$-representation. Set $k=\operatorname{dim} S W-\operatorname{dim} S W_{s}$.

(1) $Y$ is $(k-2)$-connected and $(k-1)$-simple.

(2) There is an isomorphism

$$
\Phi: H_{k-1}(Y) \rightarrow \bigoplus_{H \in \mathcal{A}} H_{k-1}\left(S\left(W^{H}\right)^{\perp}\right) \cong \bigoplus_{H \in \mathcal{A}} \mathbb{Z}
$$

by the following composite of isomorphisms:

$$
H_{k-1}(Y) \stackrel{j_{*}}{\longrightarrow} \bigoplus_{H \in \mathcal{A}} H_{k-1}\left(S W \backslash S W^{H}\right) \stackrel{\oplus i_{H *}}{\longleftarrow} \bigoplus_{H \in \mathcal{A}} H_{k-1}\left(S\left(W^{H}\right)^{\perp}\right),
$$

where $i_{H}, j$ are the inclusions.

(3) The Hurewicz homomorphism $h: \pi_{k-1}(Y) \rightarrow H_{k-1}(Y)$ is an isomorphism.

Proof. (1): Note $k \geq 2$. The $(k-2)$-connectivity follows by a general position argument. If $k>2$, then $Y$ is 1 -connected, hence $(k-1)$-simple. If $k=2$, then $\pi_{1}(Y)$ is abelian as seen below, so $Y$ is 1-simple.

(2): Set $\mathcal{B}:=\operatorname{Iso}(S W) \backslash\{1\}$. Note $Y=\bigcap_{H \in \mathcal{B}}\left(S W \backslash S W^{H}\right)$. Note also that

$$
\operatorname{dim}\left(S W^{H} \cap S W^{K}\right)=\operatorname{dim} S W^{H K} \leq \operatorname{dim} S W_{s}-2,
$$

provided $H \neq K(H, K \in \mathcal{B})$, in fact, since $\operatorname{dim} S W^{H}-\operatorname{dim} S W^{K}$ is even whenever $H \leq K$, it suffices to show $\operatorname{dim} S W^{H K}<\operatorname{dim} S W_{s}$. If $H \notin \mathcal{A}$ or $K \notin \mathcal{A}$, this is obvious. In the case where $H \in \mathcal{A}$ and $K \in \mathcal{A}$, if $\operatorname{dim} S W^{H K}=\operatorname{dim} S W_{s}$, then one can see that $S W^{H}=S W^{H K}=S W^{K}$, and hence $H=K$, which is a contradiction.

Using the Mayer-Vietoris exact sequence inductively, one can see that

$$
H_{k-1}(Y) \stackrel{j_{*}}{\longrightarrow} \bigoplus_{H \in \mathcal{B}} H_{k-1}\left(S W \backslash S W^{H}\right)
$$

is an isomorphism. Since the inclusion $i_{H}: S\left(W^{H}\right)^{\perp} \rightarrow S W \backslash S W^{H}$ is a homotopy equivalence and $\operatorname{dim} S\left(W^{H}\right)^{\perp}=\operatorname{dim} S W-\operatorname{dim} S W^{H}-1$, we have that $H_{k-1}\left(S W \backslash S W^{H}\right) \cong \mathbb{Z}$ when $\operatorname{dim} S W^{H}=\operatorname{dim} S W_{s}$, and $H_{k-1}\left(S W \backslash S W^{H}\right)=0$ when $\operatorname{dim} S W^{H}<\operatorname{dim} S W_{s}$.

(3): If $k>2$, this follows from the Hurewicz theorem. In case of $k=2$ it suffices to show that $\pi_{1}(Y)$ is abelian. Set $Y^{\prime}=S W \backslash \bigcup_{H \in \mathcal{A}} S W^{H}$. Since $\operatorname{dim} S W-$ $\operatorname{dim} S W^{K} \geq k+2$ for $1 \neq K \in \operatorname{Iso}(S W) \backslash \mathcal{A}$, the inclusion $Y \subset Y^{\prime}$ induces an isomorphism $\pi_{1}(Y) \cong \pi_{1}\left(Y^{\prime}\right)$. Moreover $Y^{\prime}$ is a deformation retract of $Z:=$ $W \backslash \bigcup_{H \in \mathcal{A}} W^{H}$. Hence it suffices to see that $\pi_{1}(Z)$ is abelian. By assumption, $\left(W^{H}\right)^{\perp}$ is 2-dimensional, and hence irreducible. Moreover $\left(W^{H}\right)^{\perp} \neq\left(W^{H^{\prime}}\right)^{\perp}$ for $H \neq H^{\prime}$, hence $W$ is decomposed as $W=\bigoplus_{H \in \mathcal{A}}\left(W^{H}\right)^{\perp} \oplus W^{\prime}$ for some $W^{\prime}$, and hence one can see that $Z$ is homeomorphic to $\prod_{H \in \mathcal{A}}\left(\left(W^{H}\right)^{\perp} \backslash\{0\}\right) \times W^{\prime}$. Thus we have $\pi_{1}(Z) \cong \bigoplus_{H \in \mathcal{A}} \mathbb{Z}$. 
We first show Theorem B in the case of type F.

Proposition 2.2. Let $M$ be of type $F$ and $S W$ an $S^{1}$-representation sphere. Suppose that

(F): $\operatorname{dim} M+1 \leq \operatorname{dim} S W-\operatorname{dim} S W^{H}$ for $1 \neq H \leq S^{1}$.

Then there exists an $S^{1}$-isovariant map from $M$ to $S W$.

Proof. Set $k=\operatorname{dim} S W-\operatorname{dim} S W_{s}$ as before. Condition (F) implies that

$$
\operatorname{dim} M+1 \leq \operatorname{dim} S W-\operatorname{dim} S W_{s}
$$

and so $k \geq 2$ and $W$ is faithful. It suffices to show the existence of an $S^{1}$-map from $M$ to $Y=S W \backslash S W_{s}$. Since $Y$ is $(k-2)$-connected and $(k-1)$-simple by Lemma 2.1 and $\operatorname{dim} M / S^{1} \leq k-2$, it follows that the obstruction groups $\mathfrak{H}_{S^{1}}^{*}\left(M ; \pi_{*-1}(Y)\right) \cong$ $H^{*}\left(M / S^{1} ; \pi_{*-1}(Y)\right)$ vanish, hence there exists an $S^{1}$-isovariant map.

Remark. In this proposition, $M$ does not need to be a rational homology sphere, however $(\mathrm{F})$ is not deduced from the existence of an isovariant map in general, for example, the projection $p: S^{1} \times M \rightarrow S^{1}$ is an $S^{1}$-isovariant map for an arbitrary manifold $M$.

A similar argument is valid in semifree case. We show:

Proposition 2.3. Let $M$ be of type $S F$ and $S W$ an $S^{1}$-representation sphere with $S^{1}$-fixed points. Suppose that

(SF): $\operatorname{dim} M \leq \operatorname{dim} S W-\operatorname{dim} S W^{H}$ for $1 \neq H \leq S^{1}$.

Then there exists an $S^{1}$-isovariant map from $M$ to $S W$.

Proof. By definition $M_{s}$ consists of isolated fixed points. Take a (small) invariant disk neighborhood $N_{i}$ at each fixed point $x_{i}$, which is $S^{1}$-diffeomorphic to some representation disk, say $D U_{i}$. Since $S^{1}$ acts semifreely on $M$, every $S U_{i}$ has a free action. Take $y \in S W^{S^{1}}$ and let $B$ be an invariant disk neighborhood of $y$ which is $S^{1}$-diffeomorphic to some $D V$. We claim that there exists an $S^{1}$-isovariant map $f_{i}: S U_{i} \rightarrow S V$. Noting that $\operatorname{dim} S U_{i}=\operatorname{dim} M-1$ and that $\operatorname{dim} S V-\operatorname{dim} S V_{s}=$ $\operatorname{dim} S W-\operatorname{dim} S W_{s}$, we have $\operatorname{dim} S U_{i}+1 \leq \operatorname{dim} S V-\operatorname{dim} S V_{s}$ by (SF). Applying Proposition 2.2 to this case, we obtain an $S^{1}$-isovariant map $f_{i}: S U_{i} \rightarrow S V$. By the radial extension, $f_{i}$ can be extended to an isovariant map $\tilde{f}_{i}: D U_{i} \rightarrow D V$, so we obtain an isovariant map $g_{i}: N_{i} \rightarrow B$ equivalent to $\tilde{f}: D U_{i} \rightarrow D V$.

The next step is to extend $g:=\coprod_{i} g_{i}: \coprod_{i} N_{i} \rightarrow B \subset S W$ to an isovariant map from $M$ to $S W$. To do this it suffices to see the existence of an $S^{1}$-map $F: X:=$ $M \backslash\left(\coprod_{i} \operatorname{int} N_{i}\right) \rightarrow Y=S W \backslash S W_{s}$ extending $g_{\mid \partial N}: \partial X \rightarrow Y$. The obstruction lies in $\mathfrak{H}_{S^{1}}^{*}\left(X, \partial X ; \pi_{*-1}(Y)\right) \cong H^{*}\left(X / S^{1}, \partial X / S^{1} ; \pi_{*-1}(Y)\right)$. Since $\operatorname{dim} X / S^{1}=$ $\operatorname{dim} M / S^{1} \leq k-1$ by condition (SF), the obstruction groups vanish, and hence there exists the required $S^{1}$-map $F$.

Remark. In this proposition, indeed, $M$ does not need to be a rational homology sphere.

\section{The multidegree And Hopf type Results}

In the case of type PF, the obstruction groups do not necessarily vanish, so we need to detect the obstruction class itself. For that purpose we shall introduce the 
multidegree and show a variant of the equivariant Hopf theorem. (See [5, II 4], 9] for the equivariant Hopf theorem.)

Throughout this section, $M$ is an orientable, connected, closed $S^{1}$-manifold of type PF and we assume that $\operatorname{Iso}(M) \subset \operatorname{Iso}(S W)$. Set $k=\operatorname{dim} S W-\operatorname{dim} S W_{s}$. We also assume that $\operatorname{dim} M-1=k$. Let $S$ be an exceptional orbit of $M$ isomorphic to $S^{1} / C, C \neq 1, S^{1}$. By the slice theorem, $S$ admits a (closed) $S^{1}$-tubular neighborhood $N$ which is $S^{1}$-diffeomorphic to $S^{1} \times_{C} D U$, where $U$ is a $k$-dimensional $C$-representation so that $C$ acts freely on $U \backslash\{0\}$. We fix an orientation of $M \backslash$ int $N$ and consider the orientation of $\partial N$ (homologically) induced as the boundary of $M \backslash \operatorname{int} N$. Taking the standard orientation of $S^{1}(\subset \mathbb{C})$, we orient $S U$ such that $\partial N \cong S^{1} \times_{C} S U$ is an orientation-preserving $S^{1}$-diffeomorphism. For simplicity we often identify $N$ [resp. $\partial N]$ with $S^{1} \times_{C} D U\left[\right.$ resp. $\left.S^{1} \times_{C} S U\right]$ hereafter.

Let $f: S^{k-1} \rightarrow Y$ be a (nonequivariant) map. Then $f$ induces the homomorphism $f_{*}: H_{k-1}\left(S^{k-1}\right) \rightarrow H_{k-1}(Y)$. Composing the isomorphism $\Phi$ in Lemma 2.1 with $f_{*}$, we have a homomorphism

$$
\Phi \circ f_{*}: H_{k-1}\left(S^{k-1}\right) \rightarrow \bigoplus_{H \in \mathcal{A}} H_{k-1}\left(S\left(W^{H}\right)^{\perp}\right)=\bigoplus_{H \in \mathcal{A}} \mathbb{Z} .
$$

(We fix orientations of $S\left(W^{H}\right)^{\perp}, H \in \mathcal{A}$, and identify $\bigoplus_{H \in \mathcal{A}} H_{k-1}\left(S\left(W^{H}\right)^{\perp}\right)$ with $\left.\bigoplus_{H \in \mathcal{A}} \mathbb{Z}.\right)$

We define $\mathrm{m}$-deg $f$, called the multidegree of $f$, by setting

$$
\mathrm{m}-\operatorname{deg} f=\Phi \circ f_{*}\left(\left[S^{k-1}\right]\right) \in \bigoplus_{H \in \mathcal{A}} \mathbb{Z},
$$

where $\left[S^{k-1}\right]$ is the fundamental class of $S^{k-1}$.

Let $f: \partial N \rightarrow Y$ be an $S^{1}$-map. Restricting $f$ to $S U$, we have a $C$-map $\bar{f}:=$ $\operatorname{Res} f: S U \rightarrow Y$. By assumption, $\operatorname{dim} S U=k-1$.

Definition. We define the multidegree m-Deg $f$ of an $S^{1}$-map $f: \partial N \rightarrow Y$ by setting

$$
\mathrm{m}-\operatorname{Deg} f:=\mathrm{m}-\operatorname{deg} \bar{f}=\Phi \circ \bar{f}_{*}([S U]) \in \bigoplus_{H \in \mathcal{A}} \mathbb{Z} .
$$

Since $N$ is orientable, m-Deg $f$ does not depend on the choice of $U$ and hence m-Deg is an $S^{1}$-homotopy invariant for $S^{1}$-maps. We fix an $S^{1}$-map $f_{0}: \partial N \rightarrow Y$. One of purposes in this section is to show the following Hopf type result.

Theorem 3.1. With the notation above,

(1) m-Deg : $[\partial N, Y]_{S^{1}} \rightarrow \bigoplus_{H \in \mathcal{A}} \mathbb{Z}$ is injective.

(2) The image of $D:=\mathrm{m}-\mathrm{Deg}-\mathrm{m}-\operatorname{Deg} f_{0}$ coincides with $\bigoplus_{H \in \mathcal{A}}|C| \mathbb{Z}$.

To show this we need some lemmas.

Lemma 3.2. The homotopy group $\pi_{k-1}(Y)$ is isomorphic to $\bigoplus_{H \in \mathcal{A}} \mathbb{Z}$, and $C$ acts trivially on $\pi_{k-1}(Y)$.

Proof. The first half follows from Lemma 2.1. The $C$-action on $\pi_{k-1}(Y)$ is coming from the $S^{1}$-action, which induces the trivial action on the homotopy group.

Lemma 3.3. The restriction $\operatorname{Res}:[\partial N, Y]_{S^{1}} \rightarrow[S U, Y]_{C}$ is a bijection.

Proof. See [5] I (4.7)]. 
Lemma 3.4. The assignment $[f] \mapsto \gamma_{C}\left(f, f_{0}\right)$, where $\gamma_{C}\left(f, f_{0}\right)$ is the obstruction to the existence of a $C$-homotopy between $f$ and $f_{0}$, gives a bijection:

$$
\rho_{C}\left(f_{0}\right):[S U, Y]_{C} \rightarrow \mathfrak{H}_{C}^{k-1}\left(S U ; \pi_{k-1}(Y)\right) .
$$

Proof. See [5, II (3.17)].

Remark. The same result holds in nonequivariant case, i.e., $\rho\left(f_{0}\right):[S U, Y] \rightarrow$ $H^{k-1}\left(S U ; \pi_{k-1}(Y)\right)$ is a bijection.

The bijection in Lemma 3.4 depends on the choice of $f_{0}$. We call $f_{0}$ the reference map.

Lemma 3.5. The forgetful maps $\varepsilon: \mathfrak{H}_{C}^{k-1}\left(S U ; \pi_{k-1}(Y)\right) \rightarrow H^{k-1}\left(S U ; \pi_{k-1}(Y)\right)$ and $\iota:[S U, Y]_{C} \rightarrow[S U, Y]$ are injective.

Proof. Set $\pi=\pi_{k-1}(Y), C_{C}^{*}=\operatorname{Hom}_{\mathbb{Z} C}\left(C_{*}(S U), \pi\right)$ and $C^{*}=\operatorname{Hom}_{\mathbb{Z}}\left(C_{*}(S U), \pi\right)$. By definition $\varepsilon$ is induced by forgetting the $C$-action. Let $\bar{\tau}: C^{k-1} \rightarrow C_{C}^{k-1}$ be the homomorphism defined by setting $\bar{\tau}(f)(c)=\sum_{g \in C} g f\left(g^{-1} c\right), f \in C^{k-1}$ and $c \in C_{k-1}$, which induces the norm homomorphism $\tau: H^{*}(S U ; \pi) \rightarrow \mathfrak{H}_{C}^{*}(S U ; \pi)$ [5. p. 123]. It is easily seen that $\tau \varepsilon$ is multiplication by $|C|$. Since $\mathfrak{H}_{C}^{k-1}(S U ; \pi) \cong$ $\pi$ is torsion free, it follows that $\varepsilon$ is injective. Moreover there is the following commutative diagram:

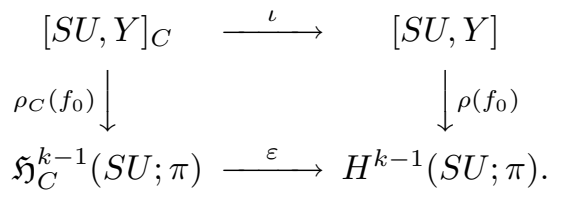

Since $\rho_{C}\left(f_{0}\right)$ and $\varepsilon$ are injective, it follows that $\iota$ is injective.

Lemma 3.6. The norm homomorphism $\tau: H^{k-1}(S U ; \pi) \rightarrow \mathfrak{H}_{C}^{k-1}(S U ; \pi)$ is an isomorphism.

Proof. Let $C_{k-1}=C_{k-1}(S U)$. Since $C_{k-1}$ is a free $\mathbb{Z} C$-module, it follows [5, II (4.5)] that $\bar{\tau}: \operatorname{Hom}_{\mathbb{Z}}\left(C_{k-1}, \pi\right) \rightarrow \operatorname{Hom}_{\mathbb{Z} C}\left(C_{k-1}, \pi\right)$ is surjective, and hence so is $\tau$. Since $H^{k-1}(S U ; \pi)$ and $\mathfrak{H}_{C}^{k-1}(S U ; \pi)$ are free abelian groups with the same rank, it follows that $\tau$ is an isomorphism.

Proof of Theorem 3.1. (1) One can see that m-Deg coincides with the following composite map:

$$
[\partial N, Y]_{S^{1}} \stackrel{\text { Res }}{\longrightarrow}[S U, Y]_{C} \stackrel{\iota}{\rightarrow}[S U, Y]=\pi_{k-1}(Y) \stackrel{h}{\cong} H_{k-1}(Y)=\bigoplus_{H \in \mathcal{A}} \mathbb{Z},
$$

which is injective by Lemmas 3.3 and 3.5

(2) To show this we first give a cohomological description of the multidegree. Let $f: \partial N \rightarrow Y$ be an $S^{1}$-map, and set $\bar{f}=\operatorname{Res} f: S U \rightarrow Y$. Using the universal 
coefficient theorem, we have the following commutative diagram:

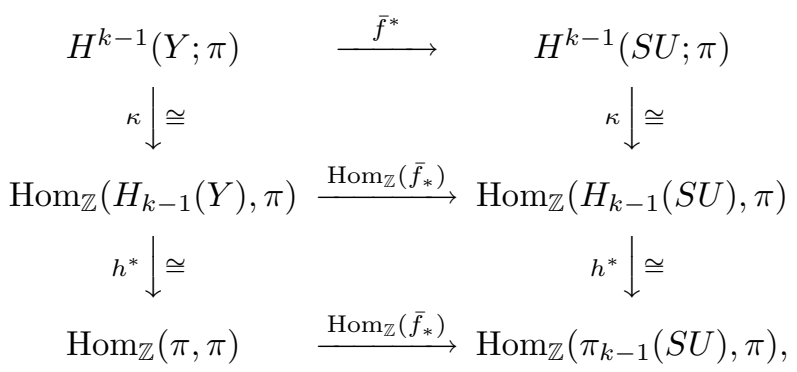

where $h$ denotes the Hurewicz homomorphism. Set

$$
\iota(Y)=\left(h^{*} \kappa\right)^{-1}\left(i d_{\pi}\right) \in H^{k-1}(Y, \pi) .
$$

Chasing the diagram, one can see that $\left\langle\bar{f}^{*} \iota(Y),[S U]\right\rangle=\bar{f}_{*}([S U]) \in \pi$, where $[S U]$ is the fundamental class in $H_{k-1}(S U ; \pi)=H_{k-1}(S U) \otimes \pi$, and hence we obtain

$$
\mathrm{m}-\operatorname{Deg} f=\Phi \circ h\left(\left\langle\bar{f}^{*} \iota(Y),[S U]\right\rangle\right) .
$$

It is well known (cf. [5, II (3.19)]) that

$$
\gamma\left(\bar{f}, \bar{f}_{0}\right)=\bar{f}^{*} \iota(Y)-\bar{f}_{0}^{*} \iota(Y) \in H^{k-1}(Y ; \pi),
$$

hence we obtain

$$
\mathrm{m}-\operatorname{Deg} f-\mathrm{m}-\operatorname{Deg} f_{0}=\Phi \circ h\left(\left\langle\gamma\left(\bar{f}, \bar{f}_{0}\right),[S U]\right\rangle\right) .
$$

Note that the image of $\varepsilon$ coincides with $|C| H^{k-1}(S U ; \pi)$, since $\tau \varepsilon$ is multiplication by $|C|$ and $\tau$ is an isomorphism by Lemma 3.6. Thus we obtain

$$
\gamma\left(\bar{f}, \bar{f}_{0}\right)=\varepsilon\left(\gamma_{C}\left(\bar{f}, \bar{f}_{0}\right)\right) \in|C| H^{k-1}(S U ; \pi),
$$

and hence

$$
D(f)=\mathrm{m}-\operatorname{Deg} f-\mathrm{m}-\operatorname{Deg} f_{0} \in \bigoplus_{H \in \mathcal{A}}|C| \mathbb{Z} .
$$

By Lemmas 3.3 and 3.4. every element of $\mathfrak{H}_{C}^{k-1}(S U ; \pi)$ is realized as $\gamma_{C}\left(\bar{f}, \bar{f}_{0}\right)$ for some $S^{1}$-map $f: \partial N \rightarrow Y$, thus we see that the image of $D$ coincides with $\bigoplus_{H \in \mathcal{A}}|C| \mathbb{Z}$.

In the rest of this section, using the multidegree, we shall discuss the extendability of $f: \partial N \rightarrow Y$ to an isovariant map from $N$ to $S W$. Take a point $y_{0} \in S W$ with isotropy group $C$, and let $V$ be the slice representation of the orbit through $y_{0}$, namely, the closed $S^{1}$-tubular neighborhood of the orbit is $S^{1}$-diffeomorphic to $S^{1} \times_{C} D V$ and $y_{0}$ corresponds to $[1,0] \in S^{1} \times_{C} D V$.

Lemma 3.7. There exists a $C$-isovariant map $\bar{g}: S U \rightarrow S V$. Moreover any $C$ isovariant map $\bar{g}: S U \rightarrow S V$ can be extended to some $S^{1}$-isovariant map $g: N \rightarrow$ $S W$.

Proof. Note that the isotropy group at each point of $S^{1} \times_{C} D V$ is a subgroup of $C$. One can see that, for any $1 \neq K \leq C$,

$$
\begin{aligned}
\operatorname{dim} S^{1} \times_{C} D U-1 & \leq \operatorname{dim} S W-\operatorname{dim} S W^{K}(\text { by } \operatorname{dim} M-1=k) \\
& =\operatorname{dim} S^{1} \times_{C} D V-\operatorname{dim}\left(S^{1} \times_{C} D V\right)^{K} \\
& =\operatorname{dim} D V-\operatorname{dim} D V^{K} .
\end{aligned}
$$


This implies that $\operatorname{dim} S U+1 \leq \operatorname{dim} S V-\operatorname{dim} S V^{K}$ for any $1 \neq K \leq C$, and hence we obtain

$$
\operatorname{dim} S U+1 \leq \operatorname{dim} S V-\operatorname{dim} S V_{s} .
$$

Then the obstruction groups $\mathfrak{H}_{C}^{*}\left(S U ; \pi_{*-1}\left(S V \backslash S V_{s}\right)\right)$ vanish, because $S V \backslash S V_{s}$ is $(l-2)$-connected and $(l-1)$-simple, $l=\operatorname{dim} S V-\operatorname{dim} S V_{s}$ (which is proved by a similar argument as in Lemma 2.1), so there exists a $C$-map from $S U$ to $S V \backslash S V_{s}$. Since $C$ acts freely on $S U$ and $S V \backslash S V_{s}$, composing the inclusion $S V \backslash S V_{s} \subset S V$, we obtain a $C$-isovariant map $\bar{g}: S U \rightarrow S V$.

By the radial extension of $\bar{g}$, we obtain a $C$-isovariant map $\tilde{g}: D U \rightarrow D V$, which induces an $S^{1}$-isovariant map $S^{1} \times_{C} \tilde{g}: S^{1} \times_{C} D U \rightarrow S^{1} \times_{C} D V$.

Remark. Under condition (PF1), this lemma still holds without the assumption $\operatorname{dim} M-1=k$.

We denote by $d_{H}(f)$ or $d_{H}(\bar{f})$ the $H$-component of m-Deg $f=\mathrm{m}$-deg $\bar{f}$.

Lemma 3.8. If an $S^{1}$-map $f: \partial N \rightarrow Y$ can be extended to an $S^{1}$-isovariant map $\tilde{f}: N \rightarrow S W$, then the $H$-component $d_{H}(f)=0$ for every $H \in \mathcal{A}$ with $H \not \leq C$.

Proof. Note that $\tilde{f}(N) \subset S W \backslash S W^{H}$, in fact, if $\tilde{f}(x) \in S W^{H}$ for some $x \in N$, then $H \leq G_{\tilde{f}(x)}=G_{x} \leq C$, which is a contradiction. Hence a $C$-map $\bar{f}=\operatorname{Res} f$ : $S U \rightarrow Y$ has the extension $\tilde{f}_{\mid D U}: D U \rightarrow S W \backslash S W^{H}\left(\simeq S\left(W^{H}\right)^{\perp}\right)$. This implies $d_{H}(f)=0$.

Conversely the extendability of $f$ to an isovariant map is detected by the multidegree. We have:

Proposition 3.9. An $S^{1}$-map $f: \partial N \rightarrow Y$ can be extended to an $S^{1}$-isovariant map from $N$ to $S W$ if and only if $d_{H}(f)=0$ for every $H \in \mathcal{A}$ with $H \not \subset C$. Moreover, for such an $S^{1}$-map $f$ and for any $a \in \bigoplus_{H \in \mathcal{A}_{C}}|C| \mathbb{Z} \subset \bigoplus_{H \in \mathcal{A}}|C| \mathbb{Z}$, where $\mathcal{A}_{C}:=\{H \in \mathcal{A} \mid H \leq C\}$, there exists an $S^{1}$-map $f^{\prime}: \partial N \rightarrow Y$ such that $f^{\prime}$ can be extended to an isovariant map and $\mathrm{m}-\operatorname{Deg} f^{\prime}=\mathrm{m}-\operatorname{Deg} f+a$.

Proof. The "only if" part has been already shown in Lemma 3.8. We show the converse. With the same notation as before, by Lemma 3.7 there is a $C$-isovariant map

$$
\bar{g}: S U \rightarrow Y^{\prime}:=S V \backslash S V_{s} \subset Y,
$$

and such a map is always extended to an $S^{1}$-isovariant map $g: \partial N \rightarrow S W$. It suffices to show that, for any $S^{1}$-isovariant map $f: \partial N \rightarrow S W$ so that $d_{H}(f)=0$ for every $H \in \mathcal{A}$ with $H \not \subset C, \bar{f}:=\operatorname{Res} f: S U \rightarrow Y$ is $C$-homotopic to some $C$-map $\bar{g}$ as above.

Let $H \in \mathcal{A}_{C}$ and let $B$ be an $H$-invariant neighborhood $H$-diffeomorphic to $D T_{y_{0}}(S W)$ a disk of the tangent space at $y_{0}$. Note $T_{y_{0}}(S W) \cong \operatorname{int} D(W-\mathbb{R})$ as $H$-representations. Choosing a small slice $D V$, one may suppose $D V \subset \operatorname{int} B$ and $D\left(V^{H}\right)^{\perp} \subset \operatorname{int}\left(B^{H}\right)^{\perp}$. Noting $\operatorname{dim} S\left(V^{H}\right)^{\perp}=\operatorname{dim} \partial\left(B^{H}\right)^{\perp}=k-1$, one can see that $\left[S\left(V^{H}\right)^{\perp}\right]=\left[\partial\left(B_{H}\right)^{\perp}\right]$ in $H_{k-1}\left(B \backslash B^{H}\right)$. Moreover, since the inclusion $\partial B \backslash \partial B^{H} \subset S W \backslash S W^{H}$ is a homotopy equivalence, it follows that $\left[\partial\left(B^{H}\right)^{\perp}\right]=$ $\left[S\left(W^{H}\right)^{\perp}\right] \in H^{k-1}\left(S W \backslash S W^{H}\right)$. Thus the inclusion $S V \backslash S V^{H} \subset S W \backslash S W^{H}$ induces an isomorphism $H_{k-1}\left(S V \backslash S V^{H}\right) \cong H_{k-1}\left(S W \backslash S W^{H}\right)$ and $\left[S\left(V^{H}\right)^{\perp}\right]$ 
maps to $\left[S\left(W^{H}\right)^{\perp}\right]$ for any $H \in \mathcal{A}_{C}$. Consequently we obtain that, for every $C$-map $\bar{g}: S U \rightarrow Y^{\prime}(\subset Y)$ and for every $H \in \mathcal{A}_{C}$,

$$
d_{H}\left(\bar{g}: S U \rightarrow Y^{\prime}\right)=d_{H}(\bar{g}: S U \rightarrow Y) .
$$

From this, fixing $\bar{g}_{0}: S U \rightarrow S V$ as in Lemma 3.7, we have the following commutative diagram:

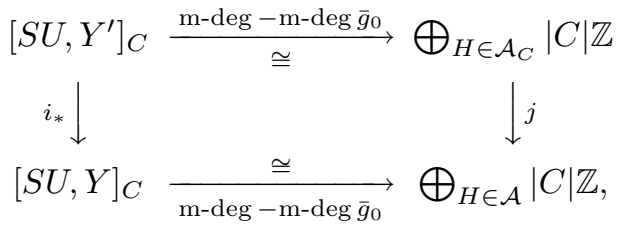

where $i: Y^{\prime} \rightarrow Y$ and $j$ are the natural inclusions. Since $d_{H}(\bar{f})=0$ for any $H \in \mathcal{A}$ with $H \not \subset C$, it follows that $[\bar{f}] \in[S U, Y]_{C}$ is in the image $i_{*}$, which completes the proof of the first half. The last half follows from Theorem 3.1 and the first half.

\section{Existence of isovariant MAPS IN TYPE PF}

Let $M$ be of type $\mathrm{PF}$ and $S W$ a representation sphere. Suppose $\operatorname{Iso}(M) \subset$ Iso $(S W)$ and conditions (PF1) and (PF2). Let $S_{i}, i=1, \ldots, t$, be the exceptional orbits with isotropy group $C_{i}$ of $M$. Let $N_{i}$ be a closed $S^{1}$-tubular neighborhood of $S_{i}$ with the slice representation $U_{i}$. By Lemma 3.7 and its remark there exists an $S^{1}$-isovariant $g_{i}: N_{i} \rightarrow S W$. Set $N=\coprod_{i} N_{i}$ and $X=M \backslash \operatorname{int} N$. Let $k=\operatorname{dim} S W-\operatorname{dim} S W_{s}$.

We shall discuss the extendability of an isovariant map $g:=\coprod_{i} g_{i}$ to an isovariant map from the whole space $M$. Set $f_{i}=g_{i \mid \partial N_{i}}: \partial N_{i} \rightarrow Y:=S W \backslash S W_{s}$ and $f=\coprod_{i} f_{i}: \partial X \rightarrow Y$. It is sufficient to see the existence of an $S^{1}$-map $F: X \rightarrow Y$ extending $f$. Conditions (PF1) and (PF2) imply that $\operatorname{dim} M-1 \leq k$, so we divide the problem into two cases:

(1) $\operatorname{dim} M-1<k$,

(2) $\operatorname{dim} M-1=k$.

In case (1) the proof is analogous to that of free or semifree case. The obstruction lies in $\mathfrak{H}_{S^{1}}^{*}\left(X, \partial X ; \pi_{*-1}(Y)\right)$, but this group vanishes since $Y$ is $(k-2)$-connected and $\operatorname{dim} X / S^{1} \leq k-1$ by assumption (1).

Next we consider the problem in case (2). The obstruction class $\gamma_{S^{1}}(f)$ to the existence of an $S^{1}$-map $X \rightarrow Y$ extending $f$ lies in $\mathfrak{H}_{S^{1}}^{k}(X, \partial X ; \pi) \cong \pi, \pi=$ $\pi_{k-1}(Y)$. Fix an $S^{1}$-map $F_{0}: X \rightarrow Y$ (which is not necessarily extending $f$ ). Note that such $F_{0}$ always exists, in fact, since $Y$ is $(k-2)$-connected and $\operatorname{dim} X / S^{1}=k$ by assumption (2), the obstruction lies in $\mathfrak{H}_{S^{1}}^{k}\left(X ; \pi_{k-1}(Y)\right)$, however, since $X$ has a (nonempty) boundary, this group also vanishes and hence there exists an $S^{1}$-map $F_{0}$. Set $f_{0}=\left.F_{0}\right|_{\partial N}: \partial N \rightarrow Y$ and $f_{0, i}=\left.F_{0}\right|_{\partial N_{i}}: \partial N_{i} \rightarrow Y$ as reference maps.

Lemma 4.1. Let $\delta: \mathfrak{H}_{S^{1}}^{k-1}(\partial X ; \pi) \rightarrow \mathfrak{H}_{S^{1}}^{k}(X, \partial X ; \pi)$ be the connecting homomorphism. Then $\delta \gamma_{S^{1}}\left(f, f_{0}\right)=\gamma_{S^{1}}(f)$.

Proof. Note [5, II (3.14)] that $\delta \gamma_{S^{1}}\left(f, f_{0}\right)=\gamma_{S^{1}}(f)-\gamma_{S^{1}}\left(f_{0}\right)$. Since $f_{0}$ has the extension $F_{0}$, it follows that $\gamma_{S^{1}}\left(f_{0}\right)=0$. 
Let $p: X \rightarrow X / S^{1}$ and $\hat{p}=\coprod p_{i}: \partial X=\coprod \partial N_{i} \rightarrow \partial X / S^{1}=\coprod \partial N_{i} / S^{1}$ be the projections. By [5, II (3.4)], these projections induce the natural isomorphisms

$$
\begin{gathered}
p^{*}: H^{k}\left(X / S^{1}, \partial X / S^{1} ; \pi\right) \rightarrow \mathfrak{H}_{S^{1}}^{k-1}(X, \partial X ; \pi), \\
\hat{p}^{*}: H^{k}\left(\partial X / S^{1} ; \pi\right) \rightarrow \mathfrak{H}_{S^{1}}^{k-1}(\partial X ; \pi) .
\end{gathered}
$$

We define isomorphisms $E: \mathfrak{H}_{S^{1}}^{k-1}(X, \partial X ; \pi) \rightarrow \pi$ and $\bar{E}: \mathfrak{H}_{S^{1}}^{k-1}(\partial X ; \pi) \rightarrow \bigoplus_{i} \pi$ by $E=e v \circ p^{*-1}$ and $\bar{E}=e v \circ \hat{p}^{*-1}$, respectively. Here $e v$ denotes the evaluation map by the fundamental class.

Proposition 4.2. Identifying $\pi$ with $\bigoplus_{H \in \mathcal{A}} \mathbb{Z}$ via $\Phi \circ h$, we have:

(1) $\bar{E}\left(\gamma_{S^{1}}\left(f, f_{0}\right)\right)=\left(\left(\mathrm{m}-\operatorname{Deg} f_{i}-\mathrm{m}-\operatorname{Deg} f_{0, i}\right) /\left|C_{i}\right|\right)_{i}$

(2) $E\left(\gamma_{S^{1}}(f)\right)=\sum_{i}\left(\mathrm{~m}-\operatorname{Deg} f_{i}-\mathrm{m}-\operatorname{Deg} f_{0, i}\right) /\left|C_{i}\right|$.

Proof. Let $r=\coprod_{i} r_{i}: \coprod_{i} S U_{i} \rightarrow \partial X=\coprod_{i} \partial N_{i}$ be the inclusion, which induces a diffeomorphism $\bar{r}=\coprod_{i} \bar{r}_{i}: \coprod_{i} S U_{i} / C_{i} \rightarrow \partial X / S^{1}=\coprod_{i} \partial N_{i} / S^{1}$. Let $\bar{p}_{i}: S U_{i} \rightarrow$ $S U_{i} / C_{i}$ be the projection. Consider the following commutative diagram:

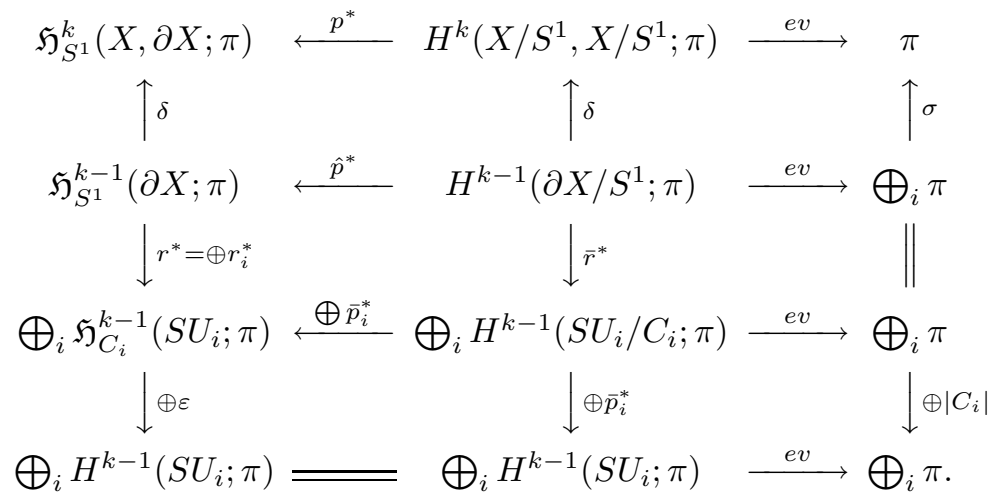

Here $\varepsilon$ is the forgetful map and $\sigma$ is defined by setting $\sigma\left(a_{i}\right)_{i}=\sum_{i} a_{i}$. Since $\bar{r}_{i}^{*}: \mathfrak{H}_{S^{1}}^{k-1}\left(\partial N_{i} ; \pi\right) \rightarrow \mathfrak{H}_{C_{i}}^{k-1}\left(S U_{i} ; \pi\right)$ maps $\gamma_{S^{1}}\left(f_{i}, f_{0, i}\right)$ to $\gamma_{C_{i}}\left(\bar{f}_{i}, \bar{f}_{0, i}\right)$, it follows from formulae (3.2) and (3.3) in section 3 that

$$
e v \circ \varepsilon \circ r_{i}^{*}\left(\gamma_{S^{1}}\left(f_{i}, f_{0, i}\right)\right)=\mathrm{m}-\operatorname{Deg} f_{i}-\mathrm{m}-\operatorname{Deg} f_{0, i} .
$$

Hence, chasing the diagram, we conclude the first assertion. By Lemma 4.1, we see that

$$
E\left(\gamma_{S^{1}}(f)\right)=E\left(\delta \gamma_{S^{1}}\left(f, f_{0}\right)\right)=\sigma \bar{E}\left(\gamma_{S^{1}}\left(f, f_{0}\right)\right) .
$$

Hence the second assertion is obtained from (1).

Corollary 4.3. An $S^{1}$-map $f: \partial X \rightarrow Y$ is extended to an $S^{1}$-map from $X$ if and only if $\sum_{i}\left(\mathrm{~m}-\operatorname{Deg} f_{i}-\mathrm{m}-\operatorname{Deg} f_{0, i}\right) /\left|C_{i}\right|=0$.

Proof. Since $E$ is an isomorphism, it follows from Proposition 4.2 that $\gamma_{S^{1}}(f)=0$ if and only if $\sum_{i}\left(\mathrm{~m}-\operatorname{Deg} f_{i}-\mathrm{m}-\operatorname{Deg} f_{0, i}\right) /\left|C_{i}\right|=0$.

We now prove the remaining case of Theorem B.

Proposition 4.4. Let $M$ be of type $P F$ and $S W$ a representation sphere. Suppose $\operatorname{Iso}(M) \subset \operatorname{Iso}(S W)$ and $\operatorname{dim} M-1=\operatorname{dim} S W-\operatorname{dim} S W_{s}(=k)$. If inequalities (PF1) and (PF2) are fulfilled, then there exists an $S^{1}$-isovariant map from $M$ to $S W$. 
Proof. It suffices to see that one can choose $S^{1}$-maps $f_{i}: \partial N_{i} \rightarrow Y$ such that every $f_{i}$ is extended to an $S^{1}$-isovariant map from $N_{i}$ to $S W$ and such that $\gamma_{S^{1}}(f)=0$ $\left(f=\coprod_{i} f_{i}\right)$. By Lemma 3.7 there are $S^{1}$-isovariant maps $g_{i}: \partial N_{i} \rightarrow S W$ extended to isovariant maps from $N_{i}$. Consider the obstruction class $\gamma_{S^{1}}(g), g=\coprod_{i} g_{i}$, which is determined by $\sum_{i}\left(\mathrm{~m}-\operatorname{Deg} g_{i}-\mathrm{m}-\operatorname{Deg} f_{0, i}\right) /\left|C_{i}\right|$. Suppose that some $H$ component of $\sum_{i}\left(\mathrm{~m}-\operatorname{Deg} g_{i}-\mathrm{m}-\operatorname{Deg} f_{0, i}\right) /\left|C_{i}\right|$ is nonzero. We note that, for any $H \in \mathcal{A}$, there exists some $C_{j} \in \operatorname{Iso}(M)$ so that $H \leq C_{j}$. In fact, if $H \not \subset C_{i}$ for every $C_{i}$, then it follows from (PF2) that $\operatorname{dim} M+1 \leq k$, which contradicts the assumption $\operatorname{dim} M-1=k$. Therefore, using Proposition 3.9. one can replace $g_{j}$ with other isovariant map $f_{j}: \partial N_{j} \rightarrow Y$ so that

$$
\sum_{i \neq j}\left(d_{H}\left(g_{i}\right)-d_{H}\left(f_{0, i}\right)\right) /\left|C_{i}\right|+\left(d_{H}\left(f_{j}\right)-d_{H}\left(f_{0, j}\right)\right) /\left|C_{j}\right|=0
$$

and $d_{K}\left(f_{j}\right)=d_{K}\left(g_{j}\right)$ for $K \neq H$. Continuing this procedure, we obtain the desired $\operatorname{map} f$.

Remark. In this proposition, it is sufficient that $M$ is an orientable closed manifold, however (PF2) is not necessarily deduced from the existence of an isovariant map. (See Example 5.2 in the next section.)

\section{Some EXAmples}

Let $T_{n}, n \in \mathbb{Z}$, denote the irreducible (complex) representation of $S^{1}$ given by $\rho_{n}: S^{1} \rightarrow S U(1)\left(=S^{1}\right), z \mapsto z^{n}$. In the following, $p, q, r$ are pairwise coprime integers greater than 1 . As a simple example of Theorem A, we have:

Example 5.1. There is no $S^{1}$-isovariant map from $S\left(T_{p} \oplus T_{1}\right)$ to $S\left(T_{p} \oplus T_{q}\right)$.

Proof. Condition (PF2) is not fulfilled (although (PF1) is fulfilled).

Remark. Obviously there exists an $S^{1}$-map $f: S\left(T_{p} \oplus T_{1}\right) \rightarrow S\left(T_{p} \oplus T_{q}\right)$, for example, $f(z, w)=\left(z, w^{q}\right) /\left\|\left(z, w^{q}\right)\right\|$ is $S^{1}$-equivariant.

Next we illustrate an example in the case when $M$ is not a rational homology sphere. Set $M=S T_{p} \times S\left(T_{1} \oplus \mathbb{R}\right)$ and $S W=S\left(T_{p} \oplus T_{q}\right)$. Note that $M$ is an $S^{1}$-manifold of type PF with two exceptional orbits whose isotropy groups are the same subgroup $\mathbb{Z}_{p}$.

Example 5.2. There exists an $S^{1}$-isovariant map $f: M \rightarrow S W$.

Proof. One can directly construct an $S^{1}$-isovariant map. Define $f: M \rightarrow S W$ by setting

$$
f(z,(w, t))=\left(z, t w^{q}\right) /\left\|\left(z, t w^{q}\right)\right\| .
$$

It is easily verified that $f$ is $S^{1}$-isovariant.

Remark. In this example, condition (PF2) is not fulfilled, however one can see that the reference map $f_{0}$ can be taken so that $d_{\mathbb{Z}_{q}}\left(f_{0,1}\right)+d_{\mathbb{Z}_{q}}\left(f_{0,2}\right)=0$. Hence the proof of Proposition 4.4 still works.

The following two examples give an answer to (an analogue of) the question posed in [16].

Example 5.3. There exists an $S^{1}$-isovariant map

$$
f: S\left(T_{p} \oplus T_{q} \oplus T_{r}\right) \rightarrow S\left(T_{1} \oplus T_{p q} \oplus T_{q r} \oplus T_{r p}\right) .
$$


Proof. Set $V=T_{p} \oplus T_{q} \oplus T_{r}$ and $W=T_{1} \oplus T_{p q} \oplus T_{q r} \oplus T_{r p}$. Note that $\operatorname{Iso}(S V) \subset$ Iso $(S W)$. Moreover, it is easily seen that $S V$ is of type PF and that both (PF1) and (PF2) are fulfilled.

Let $C=\mathbb{Z}_{n}$ be the cyclic group of order $n$. Let $g$ be a generator of $C$. The irreducible orthogonal $C$-representations $R_{i}$ are given by the following: $R_{0}=\mathbb{R}$ with trivial action, and $R_{i}$ is the 2-dimensional $C$-representation on which $g$ acts by rotation $2 \pi i / n$ for each $0<i<n / 2$. When $n$ is even, $R_{n / 2}$ is the 1 -dimensional $C$-representation on which $g$ acts antipodally. We have:

Example 5.4. There exists a $\mathbb{Z}_{p q r}$-isovariant map

$$
f: S\left(R_{p} \oplus R_{q} \oplus R_{r}\right) \rightarrow S\left(R_{1} \oplus R_{p q} \oplus R_{q r} \oplus R_{r p}\right),
$$

provided that none of $p, q, r$ are equal to 2 . Otherwise there is no $C$-isovariant map.

Proof. If none of $p, q, r$ are equal to 2 , then each $R_{i}$ is obtained by restricting the $S^{1}$-representation $T_{i}$ to the $\mathbb{Z}_{p q r}$-action, and hence restricting an $S^{1}$-isovariant map as in Example 5.3. we obtain the required map. Suppose that one of $p, q, r$ is equal to 2 , say $p=2$. Note that $\operatorname{dim} R_{q r}=1$. If there is a $\mathbb{Z}_{p q r}$-isovariant map

$$
f: S V:=S\left(R_{2} \oplus R_{q} \oplus R_{r}\right) \rightarrow S W:=S\left(R_{1} \oplus R_{2 q} \oplus R_{q r} \oplus R_{2 r}\right),
$$

it follows from Proposition 1.2 that

$$
4=\operatorname{dim} S V-\operatorname{dim} S V^{\mathbb{Z}_{2}} \leq \operatorname{dim} S W-\operatorname{dim} S W^{\mathbb{Z}_{2}}=3
$$

by regarding $f$ as a $\mathbb{Z}_{2}$-isovariant map. This is a contradiction.

\section{REFERENCES}

[1] K. Borsuk, Drei Sätze über die n-dimensionale Sphäre, Fund. Math, 20 (1933), 177-190.

[2] G. E. Bredon, Introduction to compact transformation groups, Academic Press, 1972. MR0413144(54:1265)

[3] W. Browder and F. Quinn, A surgery theory for $G$-manifolds and stratified sets, ManifoldsTokyo 1973, 27-36, Univ. Tokyo Press, Tokyo, 1975. MR0375348 (51:11543)

[4] M. Clapp and D. Puppe, Critical point theory with symmetries, J. Reine Angew. Math. 418 (1991), 1-29. MR.1111200 (92d:58031)

[5] T. tom Dieck, Transformation groups, Walte de Gruyter, Berlin, New York, 1987. MR0889050 (89c:57048)

[6] A. Dold, Simple proofs of some Borsuk-Ulam results, Proceedings of the Northwestern Homotopy Theory Conference (Evanston, Ill., 1982), 65-69, Contemp. Math., 19. MR0711043 (85e:55003)

[7] K. H. Dovermann, Almost isovariant normal maps, Amer. J. Math. 111 (1989), 851-904. MR1026286 (91b:57042)

[8] G. Dula and R. Schultz, Diagram cohomology and isovariant homotopy theory, Mem. Am. Math. Soc. 110, no. 527, 1994. MR.1209409 (95a:55028)

[9] D. Ferrario, On the equivariant Hopf theorem, Topology 42 (2003), 447-465. MR1941444 (2003i:55011)

[10] M. Furuta, Monopole equation and the 11/8-conjecture, Math. Res. Lett. 8 (2001), 279-291. MR:1839478 (2003e:57042)

[11] K. Kawakubo, The theory of transformation groups, Oxford University Press, 1991. MR:1150492 (93g:57044)

[12] W. Lück, Transformation groups and algebraic K-theory, Lecture Notes in Mathematics, 1408, Springer-Verlag, Berlin, 1989. MR:1027600 (91g:57036)

[13] J. Matoušek, Using the Borsuk-Ulam theorem. Lectures on topological methods in combinatorics and geometry, Universitext, Springer, 2003. MR.1988723 (2004i:55001) 
[14] D. Montgomery and C. T. Yang, Differentiable pseudo-free circle actions on homotopy seven spheres, Proceedings of the Second Conference on Compact Transformation Groups, Part I, 41-101, Lecture Notes in Math., Vol. 298, Springer, Berlin, 1972. MR0362383 (50:14825)

[15] I. Nagasaki, The weak isovariant Borsuk-Ulam theorem for compact Lie groups, Arch. Math. 81 (2003), 348-359. MR2013267 (2004i:55002)

[16] I. Nagasaki, Isovariant maps between representation spaces (Japanese), Transformation groups from new points of view (Japanese) (Kyoto, 2002), Sūrikaisekikenkyūsho Kōkyūroku No. 1290 (2002), 83-94. MR 1982458

[17] T. Petrie, Pseudoequivalences of $G$-manifolds, Algebraic and geometric topology, 169-210, Proc. Sympos. Pure Math., 32, 1978. MR0520505 (80e:57039)

[18] H. Steinlein, Borsuk's antipodal theorem and its generalizations and applications: a survey, Topological methods in nonlinear analysis, 166-235, Montreal, 1985. MR0801938 (86k:55002)

[19] H. Steinlein, Spheres and symmetry: Borsuk's antipodal theorem, Topol. Methods Nonlinear Anal. 1 (1993), 15-33. MR1215255 (94b:55008)

[20] A. G. Wasserman, Isovariant maps and the Borsuk-Ulam theorem, Topology Appl. 38 (1991), 155-161. MR1094548(92j:55002)

Department of Mathematics, Graduate School of Science, Osaka University, ToyONAKA, OSAKA 560-0043, JAPAN

E-mail address: nagasaki@math.sci.osaka-u.ac.jp 